\title{
M-TEST: A Test Chip for MEMS Material Property Measurement Using Electrostatically Actuated Test Structures
}

\author{
Peter M. Osterberg and Stephen D. Senturia, Fellow, IEEE
}

\begin{abstract}
A set of electrostatically actuated microelectromechanical test structures is presented that meets the emerging need for microelectromechanical systems (MEMS) process monitoring and material property measurement at the wafer level during both process development and manufacturing. When implemented as a test chip or drop-in pattern for MEMS processes, M-Test becomes analogous to the electrical MOSFET test structures (often called E-Test) used for extraction of MOS device parameters. The principle of M-Test is the electrostatic pull-in of three sets of test structures [cantilever beams (CB's), fixed-fixed beams (FB's), and clamped circular diaphragms (CD's)] followed by the extraction of two intermediate quantities (the $S$ and $B$ parameters) that depend on the product of material properties and test structure geometry. The $S$ and $B$ parameters give a direct measure of the process uniformity across an individual wafer and process repeatability between wafers and lots. The extraction of material properties (e.g., Young's modulus, plate modulus, and residual stress) from these $S$ and $B$ parameters is then accomplished using geometric metrology data. Experimental demonstration of M-Test is presented using results from MIT's dielectrically isolated wafer-bonded silicon process. This yielded silicon plate modulus results which agreed with literature values to within $\pm 4 \%$. Guidelines for adapting the method to other MEMS process technologies are presented. [204]
\end{abstract}

Index Terms - Computer-aided design for microelectromechanical systems, material properties, MEMCAD, MEMS, MEMS modeling, microactuators, microelectromechanical systems, microfabrication, micromachining, microsensors, microstructures, plate modulus, Poisson ratio, pull-in voltage, residual stress, wafer bonding, Young's modulus.

\section{INTRODUCTION}

W ITH THE growth of micromachining process technologies for microelectromechanical systems (MEMS), there has developed a need for simple, accurate, and standardized process monitoring and material property extraction capability (e.g., Young's modulus, plate modulus, and residual stress) at the wafer level during process development and

Manuscript received March 26, 1996; revised March 3, 1997. Subject Editor, R. O. Warrington. This work was supported by the Semiconductor Research Corporation under Contract 95-SC-309 and ARPA under Contract J-FBI-92-196.

P. M. Osterberg was with the Microsystems Technology Laboratories, Department of Electrical Engineering and Computer Science, Massachusetts Institute of Technology, Cambridge, MA 02139 USA. He is now with the School of Engineering, University of Portland, Portland, OR 97203 USA (email: oster@up.edu).

S. D. Senturia is with the Microsystems Technology Laboratories, Department of Electrical Engineering and Computer Science, Massachusetts Institute of Technology, Cambridge, MA 02139 USA.

Publisher Item Identifier S 1057-7157(97)04600-3. manufacturing [1]. A broad array of material property extraction methods has been proposed in the literature. These methods include a resonant frequency measurement technique on cantilever beam (CB) test structures [2], [3], a direct tensile stress measurement technique [4], a capacitance/voltage $(C / V)$ measurement technique on fixed-fixed beam (FB) bridge structures [5], the direct mechanical bending of $\mathrm{CB}$ test structures by a known force and measurement of the resulting deflection [6], a load/deflection technique on suspended thinfilm membranes under tensile stress and known pressure load [1], [4], [7], and an electrostatic pull-in approach using tethered rigid parallel-plate structures [8]. Most of the techniques require special micromachined structures or special test fixtures, which makes them difficult to use in routine wafer-level measurements, for example, in a manufacturing environment. Furthermore, the extracted material property values have not yet been demonstrated to have the accuracy required for routine use. For example, a survey by Schweitz [6] of material property measurement methods noted that the various reported Young's Modulus mean values obtained for [011] singlecrystal silicon fell between 120-220 GPa with uncertainties of $20 \%$ or higher. A reasonable goal for uncertainty should be a few percent in the extracted material property. The $20 \%$ or larger uncertainties summarized by Schweitz, together with the wide scatter of reported results, suggests that improved measurement methods are needed.

The philosophy adopted in this work is that a viable test method must be usable at the wafer level in a manufacturing environment (and, thus, must be nondestructive), require only readily available test equipment, and be supported with documented structure-design, data-acquisition and data-analysis methods, and calibrated models for quantitative interpretation of results. Out of the known methods, the best candidate for meeting these requirements was judged to be the use of electrostatic pull-in of microfabricated test structures, a method pioneered by Petersen [2] and later extended by Najafi and Suzuki [8].

Electrostatic pull-in is a well-known sharp instability in the behavior of an elastically supported structure subjected to parallel-plate electrostatic actuation. Because the event is sharp, accurate measurements of the actuation voltage required to reach pull-in can be easily made at the wafer level using standard electrical test equipment plus a microscope. However, in the work to date, the models used for data interpretation have not been sufficiently detailed to achieve the required 
accuracy in extracted material properties. Further, guidelines for structure design to match a given process and robust datareduction methods to achieve the maximum possible precision have not been previously reported. The goal of the present paper is to place the electrostatic pull-in method on a sound experimental footing so that it can be widely used in MEMS process testing and material property extraction. Accordingly, when implemented as a MEMS test chip or drop-in pattern, the method is given the name "M-Test," in analogy to the electrical MOSFET test structures (often called E-Test) used for extraction of MOS device parameters.

The M-Test concept is based on an array of microelectromechanical test structures of varying dimensions. Three specific structures are studied here (and the concept can be readily extended to a broader class of structures): CB's, FB's, and clamped circular diaphragms (CD's), all suspended above a fixed ground plane by a gap (see Fig. 1). In each case, a voltage is applied to the upper movable conductor, causing it to deflect downward toward the underlying fixed ground plane due to the electrostatic attraction. At a critical "pullin" voltage $V_{\mathrm{PI}}$, the upper conductor becomes unstable and spontaneously collapses (or pulls in) to the ground plane. The pull-in voltage is related to the test structure's geometry and intrinsic material properties. Therefore, the pull-in data provides a direct indication of the uniformity and repeatability of a given process, and when supplemented with models and geometric metrology data, the variation of pull-in voltage with device geometry can also be used to extract material properties.

This work presents: 1) the development of closed-form quantitative models for the pull-in of M-Test structures derived from two-dimensional (2-D) and three-dimensional (3-D) numerical simulations using the Massachusetts Institute of Technology's software package for the computer-aided design of microelectromechanical systems (MIT MEMCAD) [10], [11]; 2) an experimental procedure and associated data-reduction method, which removes geometrically correlated statistical variation in order to improve the precision of the results and uses geometric data on the test-structure dimensions (such as beam thickness and undeformed gap) to extract material properties from the pull-in data; and 3) experimental verification of the M-Test method using data from MIT's dielectrically isolated single-crystal silicon wafer-bonded process [12].

\section{Model Development}

This section presents the development of a quantitative closed-form model for the dependence of the pull-in voltage of a structure on its dimensions and material properties. "Closedform" in this context means an empirical fit to simulated data using a theoretically derived functional form, as will be explained later. In order for the model to be accurate, the structure must be fabricated to meet standards of ideality discussed below. It is then shown that the variation of pullin voltage with in-plane structural dimensions (beamlength or diaphragm diameter) can be efficiently expressed in terms of two intermediate quantities called $S$ and $B$ parameters. $S$ is the "stress" parameter and is equal to $\tilde{\sigma} t g_{0}^{3}$, where $\tilde{\sigma}$ is the effective residual stress, $t$ is the structure thickness, and $g_{0}$
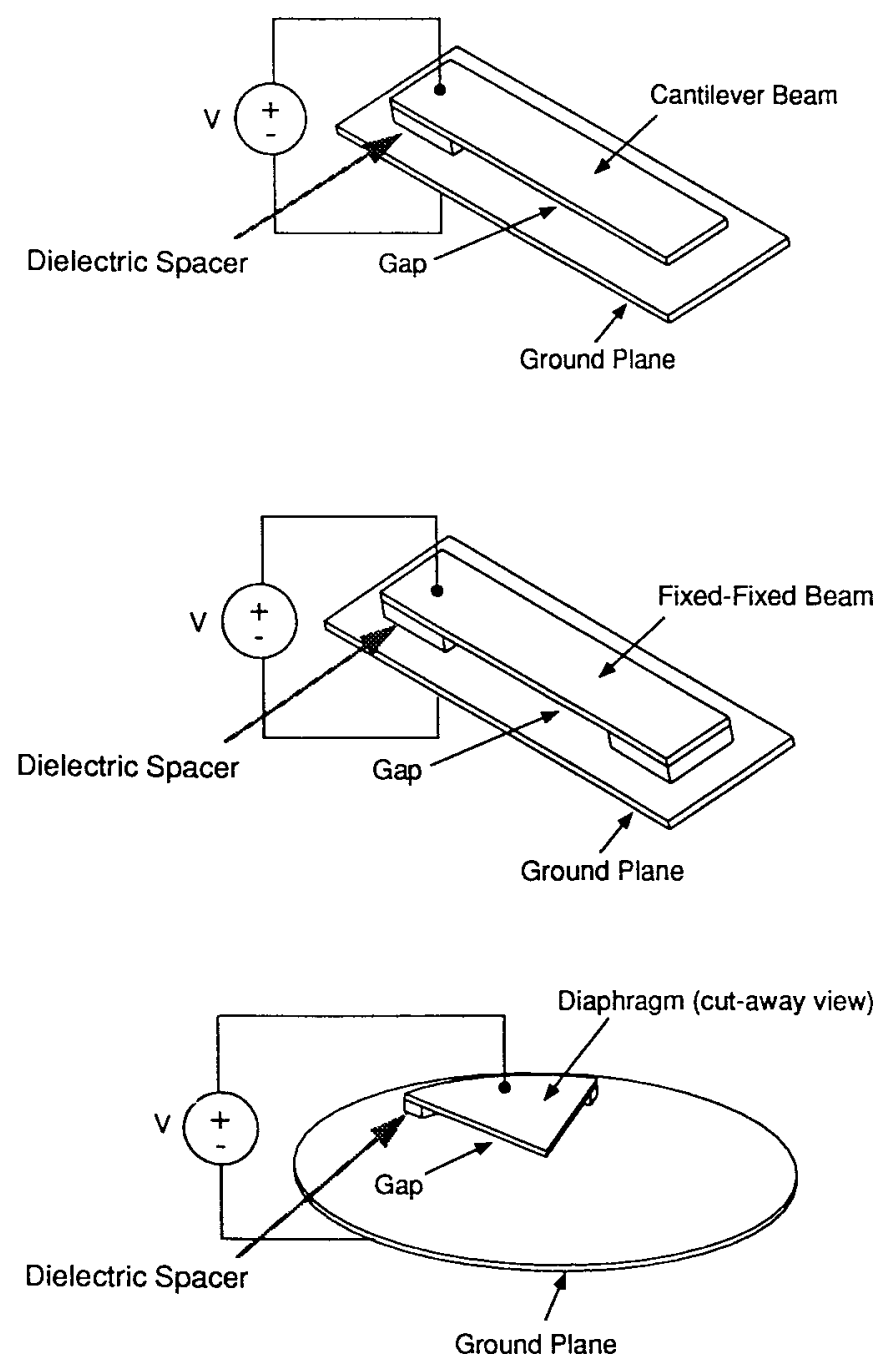

Fig. 1. Three M-Test pull-in test structures: CB, FB, and CD.

is the undeformed gap. $B$ is the "bending" parameter and is equal to $\tilde{E} t^{3} g_{0}^{3}$, where $\tilde{E}$ is the effective stiffness for the test structure. Because both $S$ and $B$ are functions of a product of material properties and test structure geometry, they provide a direct quantitative measure of overall process uniformity and repeatability (by analogy, the threshold voltage of a MOSFET depends on a combination of material properties and geometry and is routinely used for testing process uniformity and repeatability), and with independently measured data on $t$ and $g_{0}$, the material properties $\tilde{\sigma}$ and $\tilde{E}$ can be extracted from $S$ and $B$.

\section{A. Ideal Test Structure}

This section defines the ideal test structure for which the MTest models are developed. Extensions to various nonidealities are discussed in Section VI. An ideal M-Test structure meets the following requirements (see Fig. 2).

1) Two conductors: one conductor is initially flat, parallel, and movable with respect to the second conductor. The second conductor is a fixed infinite ground plane.

2) The movable conductor operates in the small-deflection regime up until pull-in (linear elastic mechanics). 


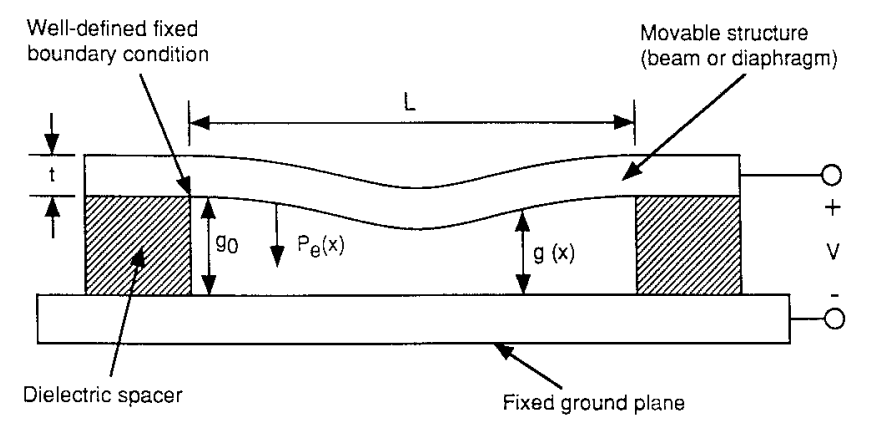

Fig. 2. Schematic cross section of the ideal generic M-Test electrostatic pull-in test structure with a suspended parallel movable structure over a fixed ground plane. An FB example is shown here. Note the position-dependent gap and electrostatic pressure, $g(x)$ and $P_{e}(x)$, respectively.

3) The movable conductor has perfect fixed boundary conditions (all six degrees of freedom at each boundary are clamped).

4) The movable conductor has a prismatic cross section, no undercutting, and no overetching.

5) The movable conductor has a negligible stress gradient (hence, no curl in the cantilevers and no buckling or bending of the beams and diaphragms).

The fixed boundary condition is created by the rigid support formed by the interface between the upper conductor and dielectric spacer (see Fig. 2). An external voltage $V$ is applied between the upper and lower conductors, which causes the upper conductor to electrostatically deflect downwards. Deflection increases with voltage until pull-in is reached.

It is assumed that FB's are fabricated by etching a film, which had an initial uniform biaxial stress $\sigma_{0}$. It is also assumed that all beams are in the Bernoulli-Euler limit, which requires that $L \gg w$ and $L \gg t$, where $w$ is the beamwidth [3]. This allows us to neglect the shear stresses near the supports and approximate the stress in the beams as purely uniaxial along the beamlength. Thus, when an FB is etched from the biaxally stressed film, the resulting residual stress is approximated as uniaxial with a uniform value of $\sigma_{0}(1-\nu)$, where $\nu$ is the Poisson ratio. When a CB is etched, the stress is fully released, except for shear stresses near the support, also assumed to be negligible. Thus, stress in the cantilevers is approximated as zero everywhere.

\section{B. Closed-Form Pull-in Models}

This section derives closed-form models for the pull-in voltage $V_{\mathrm{PI}}$ of the three ideal test structures (see Fig. 1) as functions of their geometry and material properties. The method used to develop these models is extendable to other test-structure geometries. Each model is ultimately based on detailed 3-D numerical quasi-static self-consistent simulation of the deformation of the test structure under the combined effect of linear elastic forces and nonlinear electrostatic forces, using the MIT MEMCAD system [10], [11]. However, because the M-Test structures are highly symmetric, indeed, nearly 2-D, it was determined that a much simpler 2-D finite-difference model, initially reported in [13] (with some important typographical errors, which are corrected here), was sufficiently precise in comparison with 3-D simulation to permit its use over a wide design space. Therefore, while a general test structure might require extensive 3-D numerical simulation to develop the pull-in model, in this case, we were able to use the 2-D simulation as a computational shortcut. Then, with a large virtual database of simulated pull-in voltages, we selected a functional form having the previously defined $S$ and $B$ parameters and the in-plane size (beamlength or diaphragm diameter) as the independent variables and three dimensionless adjustable parameters as fitting parameters. The resulting closed-form analytical expressions proved to be an excellent representation of the simulated data and, thus, constitute the required models.

The strategy for selecting the functional form is of interest because it guides the extension of this model to other test structures. We introduce a simplified one-dimensional (1-D) pull-in model in which the pull-in voltage depends on the undeformed gap and on the linear elastic response to an applied uniform load. While not numerically accurate, this model has the virtue of providing a functional form, which, for many structures, can be approximated analytically by solving a suitable linear differential equation. Dimensional analysis of the solution to the differential equation guides the definition of key parameters ( $S$ and $B$ in this case) and the assignment of numerical fitting parameters, which can be adjusted to take account of the nonlinearity of the electrostatic actuation and other effects, such as fringing fields. Full details of the method can be found in [13]-[15]; the key points are presented below.

1) 2-D and 3-D Simulations: The 2-D numerical simulation was based on well-known beam and plate theory [16], enhanced with a first-order fringing-field correction [14], [15]. Table I shows the governing differential equations for the 2-D simulations. The factor in parentheses on the right of the cantilever and beam equations is the fringing-field correction. For diaphragms, the effective modulus $\tilde{E}$ in Table I is $E /\left(1-\nu^{2}\right)$, where $E$ is Young's modulus. For cantilever and FB's, $\tilde{E}$ is dependent on the beamwidth [17]. A beam is considered wide when $w \geq 5 t$. Wide beams exhibit plane-strain conditions, and, therefore, $\tilde{E}$ becomes the plate modulus $E /\left(1-\nu^{2}\right)$. A beam is considered narrow when $w<5 t$. In this case, $\tilde{E}$ simply becomes the Young's modulus $E$. The effective residual stress $\tilde{\sigma}$ is the original biaxial residual stress $\sigma_{0}$ for diaphragms, while for CB's and FB's, $\tilde{\sigma}$ is zero and $\sigma_{0}(1-\nu)$, respectively, as explained earlier. The correctness of the use of the above ranges in effective modulus $\tilde{E}$ and effective residual stress $\tilde{\sigma}$ was confirmed with finite-element simulation. This model assumes only small-angle bending and neglects any nonuniformity in the electric field due to curvature. These will prove to be very reasonable approximations for the structures examined here, which are constructed with initially parallel components and have gaps that are small compared to their lateral dimensions.

A finite-difference iterative relaxation method solver was implemented in MATLAB [15], [18] to solve for the pullin voltage by observing the onset of numerical instability (the incremental stiffness of the beam goes to zero at pull-in, so the solution becomes unstable). Discretizations of 100 points were used along the axial direction for beams and along the radial 
TABLE I

Governing Differential Equations for the Three M-Test Pull-In Test Structures. $I=(1 / 12) w t^{3}, w$ Is BeAmwidth, $g=g(x)$ Is GaP, $T_{b}=\tilde{\sigma} w t, T_{d}=\tilde{\sigma} t, \varepsilon_{0}$ Is Permittivity of Vacuum, and $V$ Is Voltage

\begin{tabular}{|c|c|}
\hline Structure & Governing Differential Equation \\
\hline \hline $\begin{array}{c}\text { Cantilever Beam } \\
\text { (CB) }\end{array}$ & $\tilde{E} I \frac{d^{4} g}{d x^{4}}=-\frac{\varepsilon_{0} V^{2} w}{2 g^{2}}\left(1+0.65 \frac{g}{w}\right)$ \\
\hline $\begin{array}{c}\text { Fixed-Fixed Beam } \\
\text { (FB) }\end{array}$ & $\tilde{E} I \frac{d^{4} g}{d x^{4}}-T_{b} \frac{d^{2} g}{d x^{2}}=-\frac{\varepsilon_{0} V^{2} w}{2 g^{2}}\left(1+0.65 \frac{g}{w}\right)$ \\
\hline $\begin{array}{c}\text { Clamped Circular } \\
\text { Diaphragm (CD) }\end{array}$ & $\frac{\tilde{E} t^{3}}{12} \nabla^{4} g-T_{d} \nabla^{2} g=-\frac{\varepsilon_{0} V^{2}}{2 g^{2}}$ \\
\hline
\end{tabular}

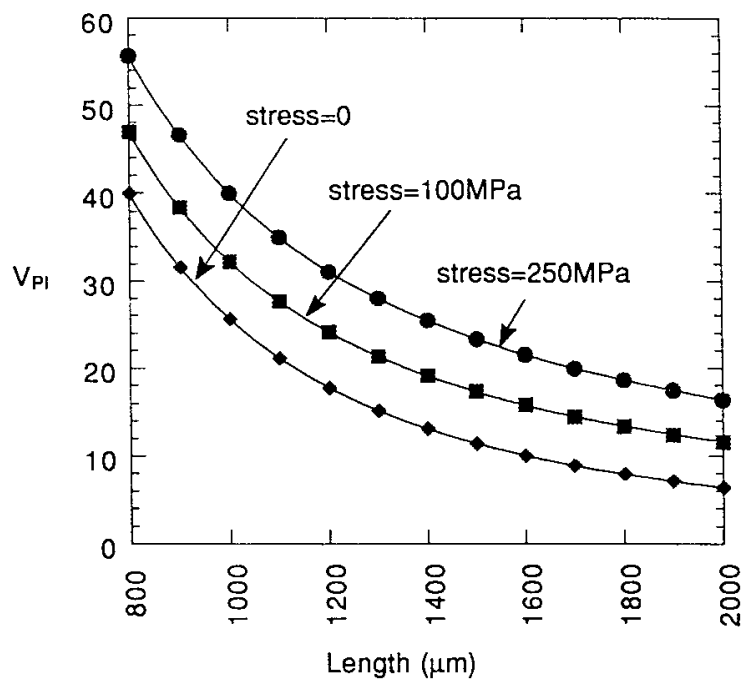

Fig. 3. Virtual database of pull-in simulation results for an FB example for three different residual stress cases. The data points are simulation results from the 2-D-distributed model. The solid lines are the closed-form model fit. In each case, beamwidth $=50 \mu \mathrm{m}$, beam thickness $=14.4 \mu \mathrm{m}$, gap $=1$ $\mu \mathrm{m}, E=169 \mathrm{GPa}$, and $\nu=0.3$. The two models agree to within $1 \%$.

direction for diaphragms. A mesh refinement study showed that pull-in voltage converged to about $1 \%$ with meshes finer than 50 points.

The accuracy of the 2-D simulation was verified by comparing six beam test cases with full 3-D MEMCAD self-consistent electromechanical simulation (see Table II). Agreement between the 2-D and 3-D MEMCAD simulations to better than $1.5 \%$ was achieved, justifying the use of the 2-D model for the extensive computations required to create the virtual database of pull-in voltage simulations. Next, to create this virtual database for CB's, FB's, and CD's, hundreds of 2-D pullin simulations were run on a large set of representative test structures with FB lengths varying from 100 to $1000 \mu \mathrm{m}, \mathrm{CB}$ lengths varying from 100 to $500 \mu \mathrm{m}$, and $\mathrm{CD}$ radii varying from 100 to $500 \mu \mathrm{m}$. In each case, the thickness was held at $14 \mu \mathrm{m}$ and the FB and CB widths were held at $50 \mu \mathrm{m}$. In addition, in each case, the Young's modulus was varied from 50 to $200 \mathrm{GPa}$, and the residual stress range was varied from 0 to $250 \mathrm{MPa}$. An example of simulated results from this virtual database for an FB case is shown as the datapoints in Fig. 3.

2) Identification of Functional Form: Fig. 4 shows a lumped 1-D pull-in model, which provides some guidance in
TABLE II

FB Pull-In Simulation Comparison. Pull-In Simulations Using Both the 2-D-Distributed Model and Mit MEMCAD Were Performed on Six FB Test Cases, Where the Beamlength and Residual Stress Were Varied. In EACH CASE, $E=169 \mathrm{GPa}, v=0.06, w=50 \mu \mathrm{m}, t=3 \mu \mathrm{m}$, and $g=1 \mu \mathrm{m}$

\begin{tabular}{|c|c|c|c|c|}
\hline Beam & Properties & \multirow{2}{*}{$\begin{array}{c}\mathrm{V}_{\mathrm{P1}} \\
\text { (2D distributed) } \\
\end{array}$} & \multirow{2}{*}{$\begin{array}{c}\mathrm{V}_{\mathrm{PI}} \\
(\mathrm{MEMCAD}) \\
\end{array}$} & \multirow[b]{2}{*}{$\Delta \%$} \\
\hline Length $(\mu \mathrm{m})$ & Stress (MPa) & & & \\
\hline 250 & 0 & 39.5 & 40.1 & $1.5 \%$ \\
\hline 250 & 100 & 56.9 & 57.6 & $1.2 \%$ \\
\hline 250 & -25 & 33.7 & 33.6 & $0.3 \%$ \\
\hline 350 & 0 & 20.2 & 20.3 & $0.5 \%$ \\
\hline 350 & 100 & 35.4 & 35.8 & $1.1 \%$ \\
\hline 350 & -25 & 13.8 & 13.7 & $0.7 \%$ \\
\hline
\end{tabular}

how the functional form is developed. The 3-D-distributed problem is approximated by a rigid body suspended by a lumped linear spring with spring constant $K_{\text {eff. The }}$ electrostatic pressure load $P_{e}$ equals $\varepsilon_{0} V^{2} /\left(2 g^{2}\right)$, where $g$ is the deformed test structure's minimum gap spacing (based on the cantilever tip deflection or beam or diaphragm center deflection) and $g_{0}$ is the undeformed gap spacing. The spring constant $K_{\text {eff }}$ has units of $\mathrm{N} / \mathrm{m}^{3}$ and is defined as $P / d_{\max }$, where $d_{\max }$ is the maximum displacement of the structure with no electrostatic load, but with a uniform-distributed pressure load $P$. The critical point is that for many structures, $K_{\text {eff }}$ can be approximated analytically. For example, the analytical 2-D solution of the uniformly loaded beam or diaphragm using the differential equations in Table I (no electrostatic load, no fringing, and only a uniform pressure load) yields

$$
K_{\mathrm{eff}}=\frac{S}{L^{2}\left[1+\frac{2\left\{1-\cosh \left(\frac{k L}{2}\right)\right\}}{\left(\frac{k L}{2}\right) \sinh \left(\frac{k L}{2}\right)}\right]}
$$

where

$$
k=\sqrt{\frac{12 S}{B}}, S=\tilde{\sigma} t g_{0}^{3}, B=\tilde{E} t^{3} g_{0}^{3}
$$

and $L$ is beamlength or diaphragm radius.

The $K_{\text {eff }}$ expression is then inserted into the classical pullin expression (corrected to first order for fringing in the case of cantilevers and beams, with the term proportional to $\gamma_{3} n$ [13], 15], where the index $n=1$ refers to cantilevers, $n=2$ to FB's, and $n=3$ to diaphragms) to yield the following analytical form for $V_{\Gamma \mathrm{I}}$ :

$$
V_{\mathrm{PI}}=\sqrt{\frac{8 K_{\mathrm{eff}} g_{0}^{3}}{27 \varepsilon_{0}\left[1+\gamma_{3 n} \frac{g_{0}}{w}\right]}} .
$$

3) Final Closed-Form Models: To create the final closedform models, the analytical expression in (2) is modified with two additional fitting parameters $\gamma_{1 n}$ and $\gamma_{2 n}$, leading to the general closed-form expression in Table III. The additional fitting parameters capture the corrections needed to allow for the fact that when the structure deflects under electrostatic actuation, the load is no longer uniform. Note that the ratio $S / B$ appears in the factor $k$, which is a measure of the relative importance of stress versus bending. The stress-dominated limit is 
TABLE III

General Closed-Form $V_{\text {Pi }}$ Expression ANd CorResPonding Bending- And Stress-Dominated Limits

\begin{tabular}{|c|c|c|}
\hline General & Bending-Dominated $(\mathrm{S} \rightarrow 0)$ & Stress-Dominated $(\mathrm{B} \rightarrow 0)$ \\
\hline \hline$\sqrt{\frac{\gamma_{1 n} S}{\varepsilon_{0} L^{2} D_{n}\left(\gamma_{2 n}, k, L\right)\left[1+\gamma_{3 n} \frac{g_{0}}{w}\right]}}$ & $\sqrt{\frac{4 \gamma_{1 n} B}{\varepsilon_{0} L^{4} \gamma_{2 n}^{2}\left(1+\gamma_{3 n} \frac{g_{0}}{w}\right)}}$ & $\sqrt{\frac{\gamma_{1 n} S}{\varepsilon_{0} L^{2}\left[1+\gamma_{3 n} \frac{g_{0}}{w}\right]}}$ \\
\hline
\end{tabular}

where:

$$
\begin{gathered}
D_{n}=1+\frac{2\left\{1-\cosh \left(\gamma_{2 n} k L / 2\right)\right\}}{\left(\gamma_{2 n} k L / 2\right) \sinh \left(\gamma_{2 n} k L / 2\right)}, k=\sqrt{\frac{12 S}{B}}, S=\tilde{\sigma} g_{0}^{3}, B=\tilde{E} t^{3} g_{0}^{3} \\
\mathrm{n}=1 \text { (CB's), } \mathrm{n}=2 \text { (FB's), } \mathrm{n}=3 \text { (CD's) }
\end{gathered}
$$

TABLE IV

Final Calculted Fitting Parameters for the CLOSED-FORM $V_{\text {PI }}$ EXPRESSIONS IN TABLE III

\begin{tabular}{|c|c|c|c|}
\hline $\begin{array}{c}\text { Numerical } \\
\text { Constants }\end{array}$ & $\begin{array}{c}\mathrm{n}=1 \\
\text { (CB's) }\end{array}$ & $\begin{array}{c}\mathrm{n}=2 \\
\text { (FB's) }\end{array}$ & $\begin{array}{c}\mathrm{n}=3 \\
\text { (CD's) }\end{array}$ \\
\hline \hline$\gamma_{l n}$ & 0.07 & 2.79 & 1.55 \\
\hline$\gamma_{2 n}$ & 1.00 & 0.97 & 1.65 \\
\hline$\gamma_{3 n}$ & 0.42 & 0.42 & 0.00 \\
\hline
\end{tabular}

$k_{L} \gg 5$, and the bending dominated limit is $k_{L} \ll 5$. Asymptotic forms for these two limits are also shown in Table III.

The final step was to solve for the fitting parameters $\left(\gamma_{1 n}, \gamma_{2 n}\right.$, and $\left.\gamma_{3 n}\right)$ using nonlinear least-squares analysis on the $\mathrm{FB}, \mathrm{CB}$, and $\mathrm{CD}$ virtual database created above, resulting in the parameters shown in Table IV. To illustrate the accuracy of the closed-form model compared to the simulated virtual database, Fig. 3 shows the closed-form model (solid curves) compared to simulated results for FB's with varying residual stress $(0,100$, and $250 \mathrm{MPa})$. Agreement is within $1 \%$.

\section{Selection of M-Test Structure Sizes}

This section describes the procedure for determining the MTest structure length and radius dimensions in order to properly characterize a particular MEMS process. (It is assumed that the thickness and gap dimensions are already set by the MEMS process in question.) M-Test structure sizing is based on an approximate analysis using the lumped pull-in model of (2). It is useful to choose CB and FB drawn mask lengths and a $\mathrm{CD}$ drawn mask radii so that the expected experimental pull-in voltages of all the M-Test structures, after processing, will fall in the $5-100-\mathrm{V}$ range, since this is the voltage range dictated by a typical M-Test experimental setup. To insure enough pull-in data, we recommend a range of at least eight different uniformly spaced length or radius dimensions of each of the three test structures with expected pull-in voltages between 5-100 V. Furthermore, it is desirable to choose a range of test structure sizes which will bracket the expected extracted bending and stress values for the process under test (referred to as a "centered" test-structure design). A centered design will optimize the accuracy and dynamic range for extraction of both the bending and stress parameters. An "off-center" design, however, will sacrifice accuracy and dynamic range of one of either the bending or stress value in exchange for improved accuracy of the other. A centered design may or may not be possible in a given process, depending on the relative sizes of the bending and stress terms. Problems such as stiction or residual compressive stress and/or stress gradients may make the larger-dimensioned structures unfeasible.

Choosing the test structure beamlengths and diaphragm radii can be done by combining the appropriate lumped model (2) with estimates for the expected Young's modulus or plate modulus and residual stress. The first step is to use the material property estimates and solve (2) for the CB and FB lengths $L$ and the $\mathrm{CD}$ radii $R$, which would yield a uniform spacing of test structure dimensions with resulting $V_{\mathrm{PI}}$ 's in the $5-100-\mathrm{V}$ range. The results are shown in Table $\mathrm{V}$, with the fringing term omitted, which is an acceptable approximation in this design phase.

Next, we determine the design center, $L_{\text {center }}$ and $R_{\text {center }}$, for the FB's and CD's, respectively, which are the values when the magnitude of both the bending- and stress-effective springforce components (i.e., $K_{\text {eff }}$ bending and $K_{\text {eff }}$ stress) are equal. For example, using an approximate analysis based on the 1-D lumped model [15], in the FB case, this occurs when

$$
\frac{32 \tilde{E} t^{3}}{L_{\text {center }}^{4}}=\frac{8 \tilde{\sigma} t}{L_{\text {center }}^{2}} .
$$

Using this approach for both $\mathrm{FB}$ and $\mathrm{CD}$, we get the values of $L_{\text {center }}$ and $R_{\text {center }}$ in Table VI. An optimal centered design would have both $L_{\text {center }}$ and $R_{\text {center }}$ fall right in the center of the chosen range of the FB and CD dimensions. But, in general, $L_{\text {center }}$ and $R_{\text {center }}$ may have to be offset, which will cause the test structure set to be shifted toward the bendingdominated or stress-dominated regime, thereby reducing the sensitivity to the other parameter. Based on experience to date, the minimum resolvable bending value $\tilde{E}_{\text {min }}$ occurs when $L_{\text {center }}$ or $R_{\text {center }}$ are about equal to the minimum chosen FB length $L_{\min }$ or CD radius $R_{\min }$, and the minimum resolvable stress value $\tilde{\sigma}_{\min }$ occurs when $L_{\text {center }}$ or $R_{\text {center }}$ are about equal to the maximum FB length $L_{\max }$ or CD radius $R_{\max }$. 
TABLE V

M-Test Length and Radius Sizing Equations for CB's, FB's, and CD's

\begin{tabular}{|c|c|c|c|c|}
\hline CB & & FB & & $\mathrm{CD}$ \\
\hline \multirow{2}{*}{$L=\sqrt[4]{\frac{16 \tilde{E} t^{3} g^{3}}{81 \varepsilon_{0} V_{P l}^{2}}}$} & \multirow{2}{*}{$L=$} & $8 \tilde{\sigma} t+\sqrt{64 \tilde{\sigma}^{2} t^{2}+\frac{432 \varepsilon_{0} \tilde{E}^{3} V_{P I}^{2}}{g_{0}^{3}}}$ & \multirow{2}{*}{$R=$} & $4 \tilde{\sigma}+\sqrt{16 \tilde{\sigma}^{2} t^{2}+\frac{72 \varepsilon_{0} \tilde{E} t^{3} V_{P I}^{2}}{g_{0}^{3}}}$ \\
\hline & & $\frac{27 \varepsilon_{0} V_{P I}^{2}}{4 g_{0}^{3}}$ & & $\frac{27 \varepsilon_{0} V_{P I}^{2}}{4 g_{0}^{3}}$ \\
\hline
\end{tabular}

TABLE VI

EXPRESSIONS FOR THE "CENTER-DESIGN" DIMENSION AND Minimum Resolvable Effective Young's Modulus and Residual Stress for both the FB and CD Test Structures

\begin{tabular}{|c|c|c|}
\hline & $\mathrm{FB}$ & $\mathrm{CD}$ \\
\hline \hline$L_{\text {center }}$ or $R_{\text {center }}$ & $\sqrt{\frac{4 \tilde{E} t^{2}}{\tilde{\sigma}}}$ & $\sqrt{\frac{4 \tilde{E} t^{2}}{3 \tilde{\sigma}}}$ \\
\hline$\tilde{E}_{\min }$ & $\frac{\tilde{\sigma} L_{\min }^{2}}{4 t^{2}}$ & $\frac{3 \tilde{\sigma} R_{\min }^{2}}{4 t^{2}}$ \\
\hline$\tilde{\sigma}_{\min }$ & $\frac{4 \tilde{E} t^{2}}{L_{\max }^{2}}$ & $\frac{4 \tilde{E} t^{2}}{3 R_{\max }^{2}}$ \\
\hline
\end{tabular}

Therefore, using the "center design" expressions from Table VI and substituting $L_{\min }$ (or $R_{\min }$ ) and $L_{\max }$ (or $R_{\max }$ ) for $L_{\text {center }}$ and $R_{\text {center, }}$, respectively, we get the resulting expressions for the minimum resolvable bending and stress, $\tilde{E}_{\text {min }}$ and $\tilde{\sigma}_{\text {min }}$, shown in Table VI.

As an example, we calculate the minimum resolvable bending and stress values $\tilde{E}_{\min }$ and $\tilde{\sigma}_{\text {min }}$ for the FB's and CD's in the particular M-Test design to be presented in Section V. In this case, the expected effective Young's modulus is approximately $170 \mathrm{GPa}$, and the expected residual stress is in the range of 1 to $10 \mathrm{MPa}$. The expected thickness and gap dimensions, after processing, are approximately 3 and 1 $\mu \mathrm{m}$, respectively. First, using the equations in Table $\mathrm{V}$, we were able to solve for the desired mask lengths and radii for all the test beams and diaphragms. Next, using the equations in Table VI, we can solve for the resulting $\tilde{E}_{\min }$ and $\tilde{\sigma}_{\text {min }}$. For FB's, we get $\tilde{E}_{\min }=11 \mathrm{GPa}$ and $\tilde{\sigma}_{\min }=25 \mathrm{MPa}$. For CD's, we get $\tilde{E}_{\min }=16 \mathrm{GPa}$ and $\tilde{\sigma}_{\text {min }}=9 \mathrm{MPa}$. In both cases, the minimum resolvable bending value is well below the expected value $(170 \mathrm{GPa})$, indicating that this value can be extracted with high accuracy. However, the minimum resolvable stress value is on the same order as our expected value $(1-10 \mathrm{MPa})$, indicating that this parameter is right at the threshold of resolution, and, therefore, its accuracy will be low. This is a bending-dominated design for the expected material properties, which was appropriate in this case because the expected residual stress was quite small.

\section{EXPERIMENTAL PROCEDURE}

The two-part M-Test experimental test procedure is outlined in Fig. 5. The first part is the measurement of pull-in and the extraction of $S$ and $B$ parameters, which are the fundamental quantitative measures of process uniformity. The second part is the extraction of material properties from these $S$ and $B$ parameters using metrology data.

\section{A. $S$ and $B$ Parameter Measurement}

1) In-Plane and Flatness Metrology: Two on-wafer measurements are required to begin the procedure: 1) average process offsets $\delta L$ and $\delta R$ between drawn and as-fabricated beamlengths and diaphragm radii and 2) verification of flatness of the structures to $\pm 0.1 \mu \mathrm{m}$.

The $\delta L$ and $\delta R$ measurements are necessary to eliminate systematic errors in the extracted $S$ and $B$ parameters. In our work, this measurement is made using a microscope (Nikon X-Y Measurescope UM-2) with a calibrated $x-y$ stage, yielding an in-plane length measurement accuracy of about $\pm 1 \mu \mathrm{m}$. This accuracy would not be sufficient for electronic device offset measurements, but is quite sufficient for typical mechanical structures, which tend to be large.

The flatness measurement is necessary since both the $S$ and $B$ parameters depend on the cube of the gap dimension and, therefore, variations in this original gap due to buckling or deflection (i.e., relative flatness) will be very important. The flatness measurement can be performed optically using an interference objective on the same microscope. A lowpass optical filter at $500 \mathrm{~nm}$ or smaller is used with a $20 \times$ objective. Fringe curvature is used to estimate flatness. If only the cantilevers are found insufficiently flat, this indicates the existence of a stress gradient, and, therefore, the CB pull-in models in this paper do not apply, but the FB's and CD's may still be used. If either the FB's or CD's are found insufficiently flat, then, this indicates the existence of either excessive compressive stress (e.g., near or at the onset of buckling) or plastic deformation, in which case one would expect large errors from the models presented here. Extension of the methods to cases with compressive stress is discussed in Section VI.

2) Pull-In Measurements: The next step is the measure-

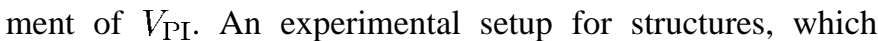
make Ohmic contact at pull-in, is shown in Fig. 6. If the structures have an insulating film between the two electrodes, Ohmic contact cannot be used, and either direct visual observation or $C / V$ measurements are needed to detect pull-in. For the devices studied here, $V_{\mathrm{PI}}$ is measured on a Wentworth Labs probe station with a Hewlett-Packard HP4145B Semiconductor Parametric Analyzer configured in a "forcevoltage/measure-current" mode. The HP4145B is programmed to slowly ramp the voltage on each test structure at $1 \mathrm{~V} / \mathrm{s}$ over a specified voltage range until pull-in is detected on the HP4145B I/V screen output interface as a sudden step in the current (see typical data in Fig. 7). For the particular beam 


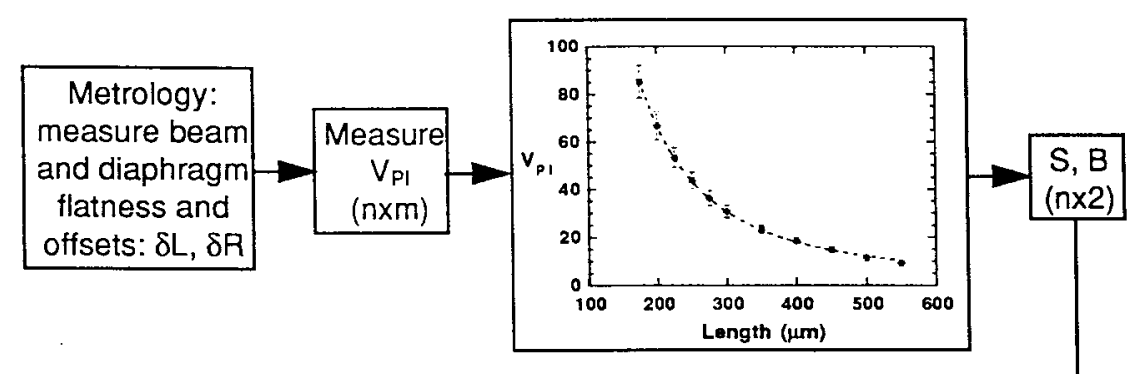

Part 1: S and B parameter measurement

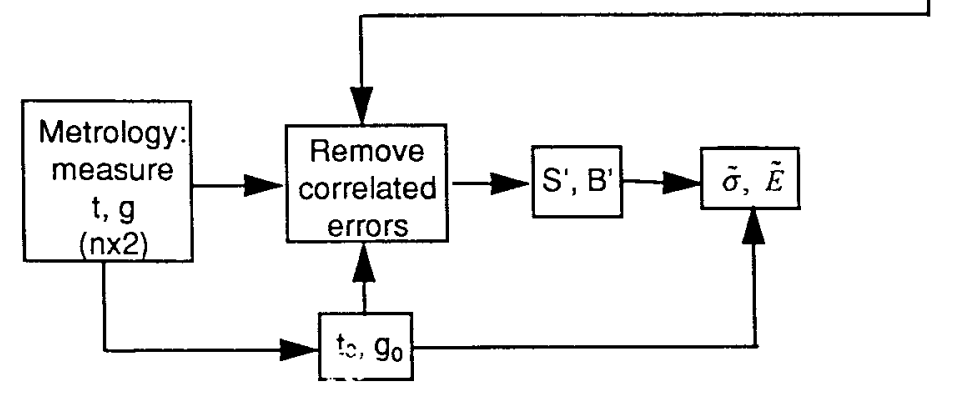

Part 2: Material property extraction

Fig. 5. M-Test experimental test procedure block diagram (two parts), where $n=\#$ of die tested per wafer and $m=\#$ of pull-in measurements per die.

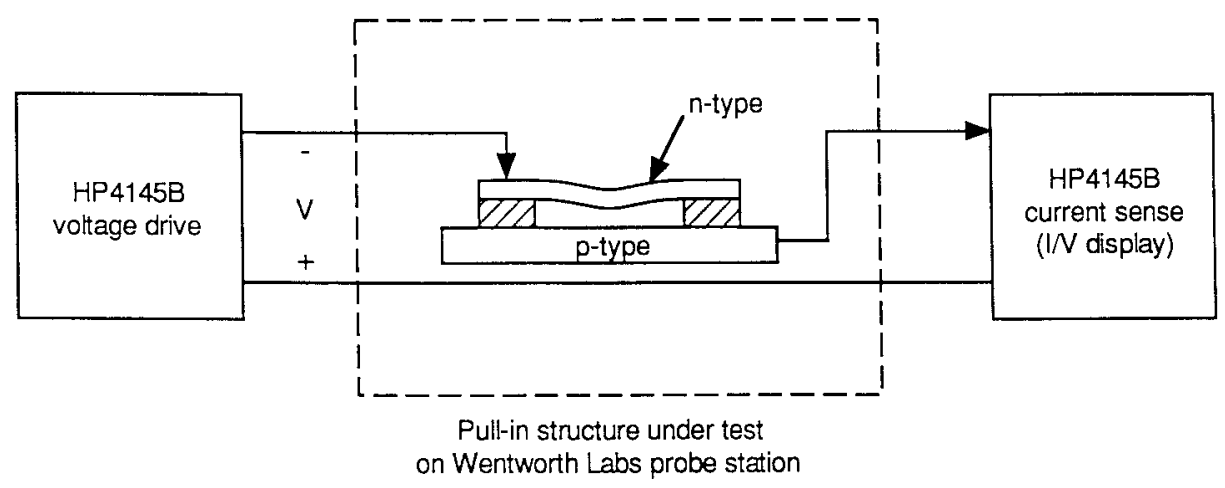

Fig. 6. M-Test pull-in measurement experimental setup. The polarity of the applied voltage in these experiments is reversed from Fig. 2 to achieve accumulation of both semiconductor surfaces. Since forces depend on the square of the applied voltage, the pull-in voltage is independent of the polarity of applied voltage, provided there are no complications from the depletion layers.

and diaphragm test structures used in this work (described in Section V), the upper movable conductor is $n$-type material, while the underlying fixed ground plane is $p$-type. The measurement must be made with the moving conductor negative so that accumulation layers form on the semiconductor surfaces. If depletion of either conductor occurs, then a correction to the measured $V_{\mathrm{PI}}$ would be needed to account for the field penetration into the semiconductor. Because the onset of pullin is very sharp, the accuracy of the measurement is determined by the voltage-ramp step increment, which was $100 \mathrm{mV}$ in our experiments. The ramp speed of $1 \mathrm{~V} / \mathrm{s}$ is slow enough to insure no dynamic effects during the bending of the test structures.

3) $S$ and $B$ Parameter Extraction: The next step is to vary $S$ and $B$ to fit the $V_{\text {PI }}$ versus $L$ (or $R$ ) data for each test structure type on each die to the closed-form model (Tables III and IV) using the $L$ and $R$ drawn mask dimensions modified by the previously measured offsets (but, of course, $S$ is zero for a $\mathrm{CB}$ ). We have used the nonlinear least squares curve fit in Kaleidagraph [19] for this purpose. An example $V_{P I}$ versus $L$ plot and corresponding curve fit is shown in Fig. 8 for two sets of FB's, one oriented along the [011] direction in a $\langle 100\rangle$ plane of single-crystal silicon, the other oriented along the [010] direction.

The die-to-die variation of $S$ and $B$ parameters across a wafer provides an immediate test of process uniformity, and the statistical ensemble of $S$ and $B$ data from a wafer provides a test of wafer-to-wafer and lot-to-lot repeatability without the necessity of any further data reduction. However, typically, one 


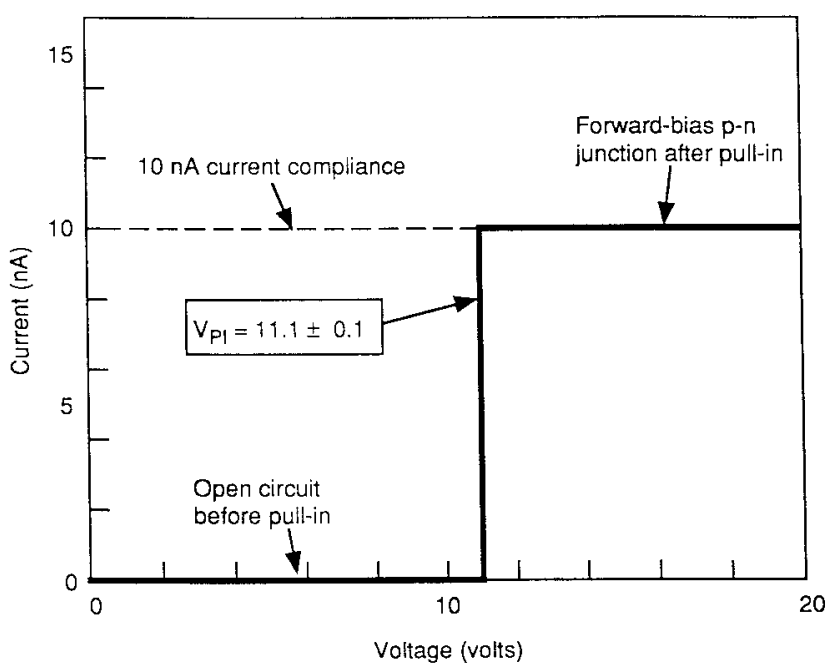

Fig. 7. Example M-Test pull-in measurement using I/V output on HP4145B. This example shows the pull-in measurement for a $500-\mu \mathrm{m}$-long, $50-\mu \mathrm{m}$-wide FB from MTEST-03. The forward-bias contact current is sensed when the $n$-type Si beam comes in contact with the $p$-type substrate at the moment of pull-in. The current compliance on the HP4145B is set to $10 \mathrm{nA}$.

is also interested in material property extraction. The suggested procedure follows.

\section{B. Material Property Extraction}

To extract material properties from the $S$ and $B$ parameters, additional metrology is required: 1) beam and diaphragm thicknesses $t$, preferably measured on each die and 2) spacer thickness or undeformed gap $g_{0}$, also preferably measured on each die. These quantities are needed; first, to remove systematic errors from the $S$ and $B$ parameters and second, for extracting the final material properties from these errorcorrected values.

1) Thickness and Gap Metrology: In this work, thickness measurements were made on each die using a Dektak II Surface Profilometer, providing accuracy of $\pm 0.01 \mu \mathrm{m}$. The oxide spacer thickness was measured ellipsometrically and should correspond to the gap dimension for an oxide-isolated silicon-wafer-bonded process. Unfortunately, die-by-die test sites for oxide thickness were not available in the mask design (but should be in future designs), so the value used was a sample average provided by the sample supplier, with an estimated accuracy of $\pm 0.01 \mu \mathrm{m}$.

2) Remove Correlated Errors in $S$ and B: Because $S$ and $B$ depend on thickness and gap, some of the observed variation in these quantities can be attributed directly to die-to-die variations in geometry. To eliminate these correlated errors, we plot the individual $S$ and $B$ parameter values versus thickness for each die (and versus gap, if available for each die) and determine if any correlation exists. We then scale the raw $S$ and $B$ values to new values, $S^{\prime}$ and $B^{\prime}$, which are the values they would have if their thickness (and gap) was exactly the global mean thickness $t_{0}$ (and the global mean gap $g_{0}$ ). We then compute averages of these scaled parameters, referred to as $S_{0}^{\prime}$ and $B_{0}^{\prime}$, respectively. This is illustrated in Fig. 9 for the data corresponding to the structures of Fig. 8.

\section{EXPERIMENTAL RESULTS}

A specific M-Test mask set (MTEST) was designed for use with MIT's dielectrically isolated wafer-bonded process [12], [15]. Fabrication details are presented, followed by complete experimental results from one test wafer (MTEST-03) out of a lot of three wafers fabricated for this purpose.

\section{A. Test-Wafer Description}

The test structures described earlier were incorporated into a mask set called MTEST, designed for the MIT dielectrically isolated wafer-bonded process [12] by Charles Hsu of MIT. MTEST is an array of three test chips alternating across a full wafer, one each for CB's, FB's, and CD's [15]. The mask layout for the FB chip is shown in Fig. 10. The CB and CD chips are not shown, but are similar to FB's in terms of a range of sizes of otherwise identical devices. Notice that the beamlengths, widths, and crystal orientations are varied. When built in a $\langle 100\rangle$ oriented wafer-bonded process, the two sets of beams are oriented along [011] and [010]. The full 4-in wafer contains 20 die, each from CB, FB, and CD for a total of 60 die.

The cross section of the typical MTEST test structure can be described by referring to Fig. 2. The upper conductor is made of $n$-type single-crystal silicon. The underlying fixed ground conductor is made of $p$-type silicon. An intermediate silicon dioxide spacer layer forms the gap. In this process, the silicon material is wafer-bonded on top of the patterned oxide, then, thinned to a specified thickness, and, finally, patterned and etched to create the final movable structures. This process is particularly well-suited for the construction of idealized pullin test structures for three reasons. First, the gap dimension is well controlled, since it is formed by etching a thermal oxide with good selectivity. Second, the fixed boundary condition at the ends of the structures approximates an ideal fixed boundary condition due to the rigidity of the oxide support. Third, there is no extraneous insulating dielectric coating on either the upper movable electrode or the underlying ground plane allowing for Ohmic contact at the moment of pull-in. This allows for a simple accurate low-noise electrical pull-in voltage measurement.

In the results presented below, only data from the wider FB and CB beams $(w=50 \mu \mathrm{m})$ from the MTEST-03 wafer are reported. The narrow beams $(w=10 \mu \mathrm{m})$ were found to be significantly altered due to a plasma overetch, which perturbed the surface of the test structure's upper movable beam and underlying fixed ground plane, requiring the use of corrections to the formulas presented here. The results from the narrow beams, including the necessary adjustments to the model, have already been presented in [20].

\section{B. FB Results}

The procedures follow those specified in Section IV, step by step.

1) Metrology: A beam-length mask bias offset measurement $(\delta L)$ was made on one MTEST-03 die inside the sweet spot. The mask had been originally designed to anticipate a $+2-\mu \mathrm{m}$ offset due to overetching of the oxide gap during 
[011] FB'S

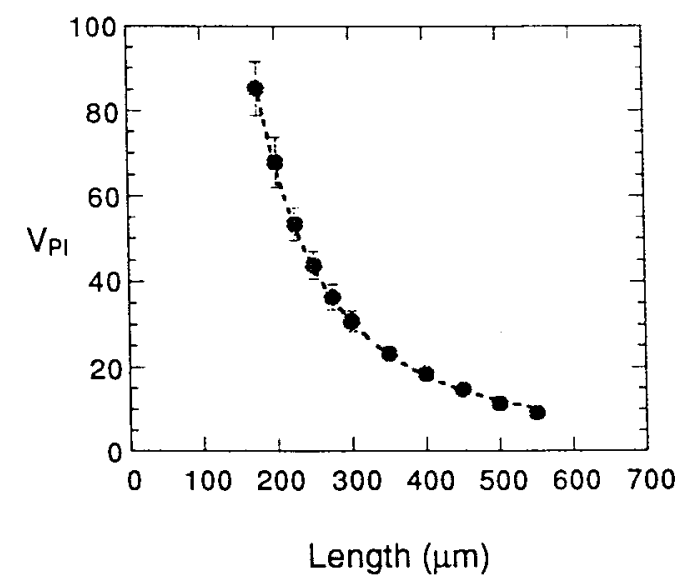

[010] FB's

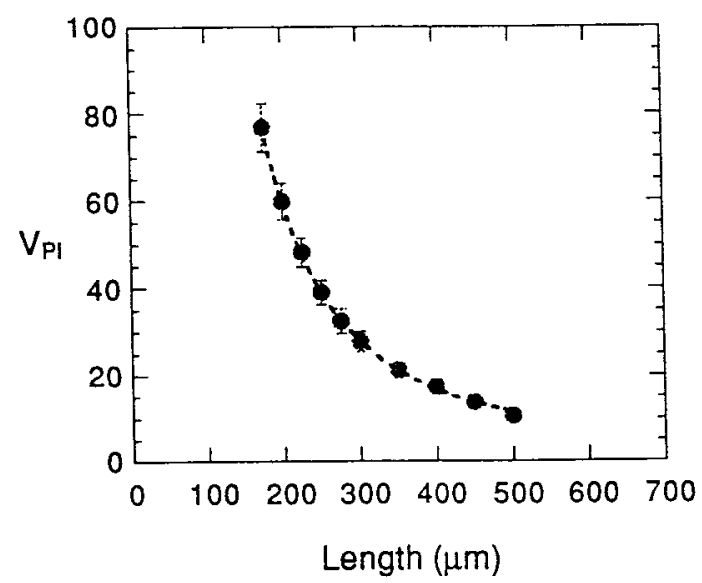

Fig. 8. $\quad V_{\mathrm{PI}}$ versus $L$ pull-in data for both [011] and [010] FB's from each of the seven FB die from MTEST-03. For simplicity, only the composite mean and standard deviation $V_{\mathrm{PI}}$ data for each beamlength from the seven die are shown.

\section{[011] FB's}

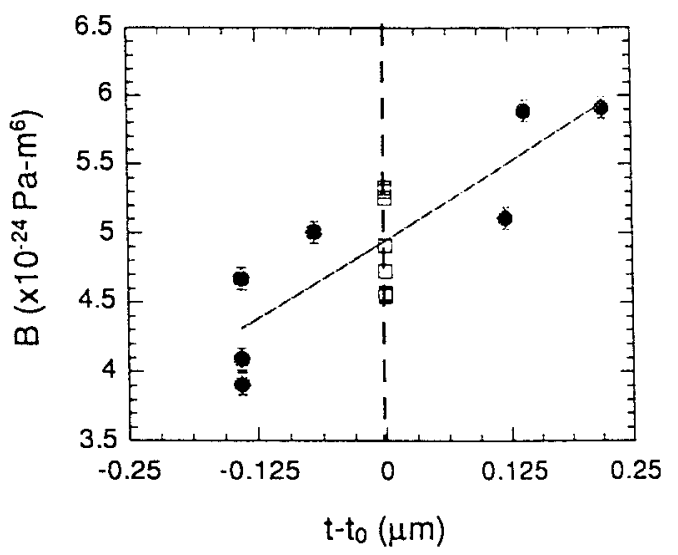

[010] FB's

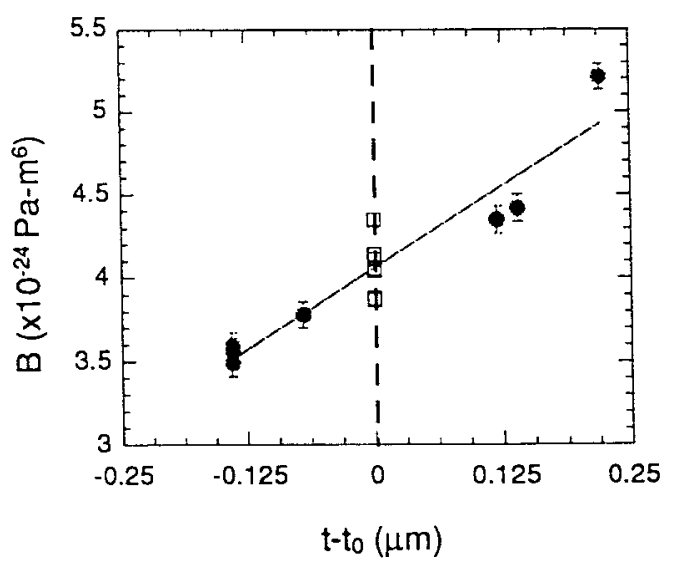

Fig. 9. Plots of $B$ parameter versus thickness $\left(t-t_{0}\right)$ for both the [011] and [010] FB's from the seven FB die locations on MTEST-03. The good correlation is clearly evident. The squares are the updated $B$ values, which have been shifted to $t_{0}$, based on the correlation. Error bars were calculated from $\delta B=(\delta B / \delta t) \delta t$.

processing; hence, the as-drawn dimensions for all but the [010] beams were intentionally preoffset from the nominal design dimensions by $-2 \mu \mathrm{m}$. The $\delta L$ measurement results showed no additional offsets between the nominal and on-chip dimension for any of the [011] FB lengths, indicating that the anticipated $+2-\mu \mathrm{m}$ offset did occur during the process, and a $+2-\mu \mathrm{m}$ offset was found for the [010] FB lengths, which had not been preoffset during mask layout. These adjustments to nominal lengths were used for subsequent data analysis.

Dektak II surface profilometry thickness measurements were made on a sample of FB's from each of the seven FB die positions inside the sweet spot of MTEST-03. The thickness mean and standard error, which includes both die-to-die variation and random measurement error, were determined to be $t_{0}=2.94 \pm 0.07 \mu \mathrm{m}$. The undeformed gap measurement $g_{0}$ was supplied by the manufacturer of the bonded wafers, based on ellipsometric measurements at five standard die locations (center, top, bottom, left, and right). The undeformed gap mean and standard error were determined to be $g_{0}=1.05 \pm 0.01 \mu \mathrm{m}$. Interferometric measurements were made on a small sample of beams from the MTEST-03 wafer. Flatness was confirmed to be better than $\pm 0.1 \mu \mathrm{m}$ as required.

2) Pull-In Measurement and Data Analysis: The ( $\left.V_{\mathrm{PI}}, L\right)$ pull-in data was taken on each of the wide [011] and [010] crystal-oriented FB's (ten data points per die using the ten FB lengths shown in Fig. 8 and seven die inside the sweet spot). Then, this pull-in data was fitted in Kaleidagraph to the closedform model using the corrected beamlengths $\left(L_{\text {drawn }}+\delta L\right.$ [011] and $L_{\text {drawn }}+\delta L$ [010]) in Kaleidagraph (see Fig. 8). The extracted $S$ and $B$ parameters from each of seven die were then plotted against the thickness value from each die, and the $S$ and $B$ values were then scaled to the nominal $t_{0}$ and $g_{0}$ values (see Fig. 9), yielding a scaled set of seven parameters, $S^{\prime}$ and $B^{\prime}(S$ and $B$ correction due to gap correlation was not done, as stated earlier). Studies of the narrow beams, which were done by Gupta et al. [20], revealed some nonideal geometry because of an overetch during fabrication. Therefore, the narrow beams were not included in this study.

The extracted parameter results are summarized in Table VII. $\bar{S}^{\prime}$ and $\bar{B}$ are the averages of the seven $S$ and $B$ values 


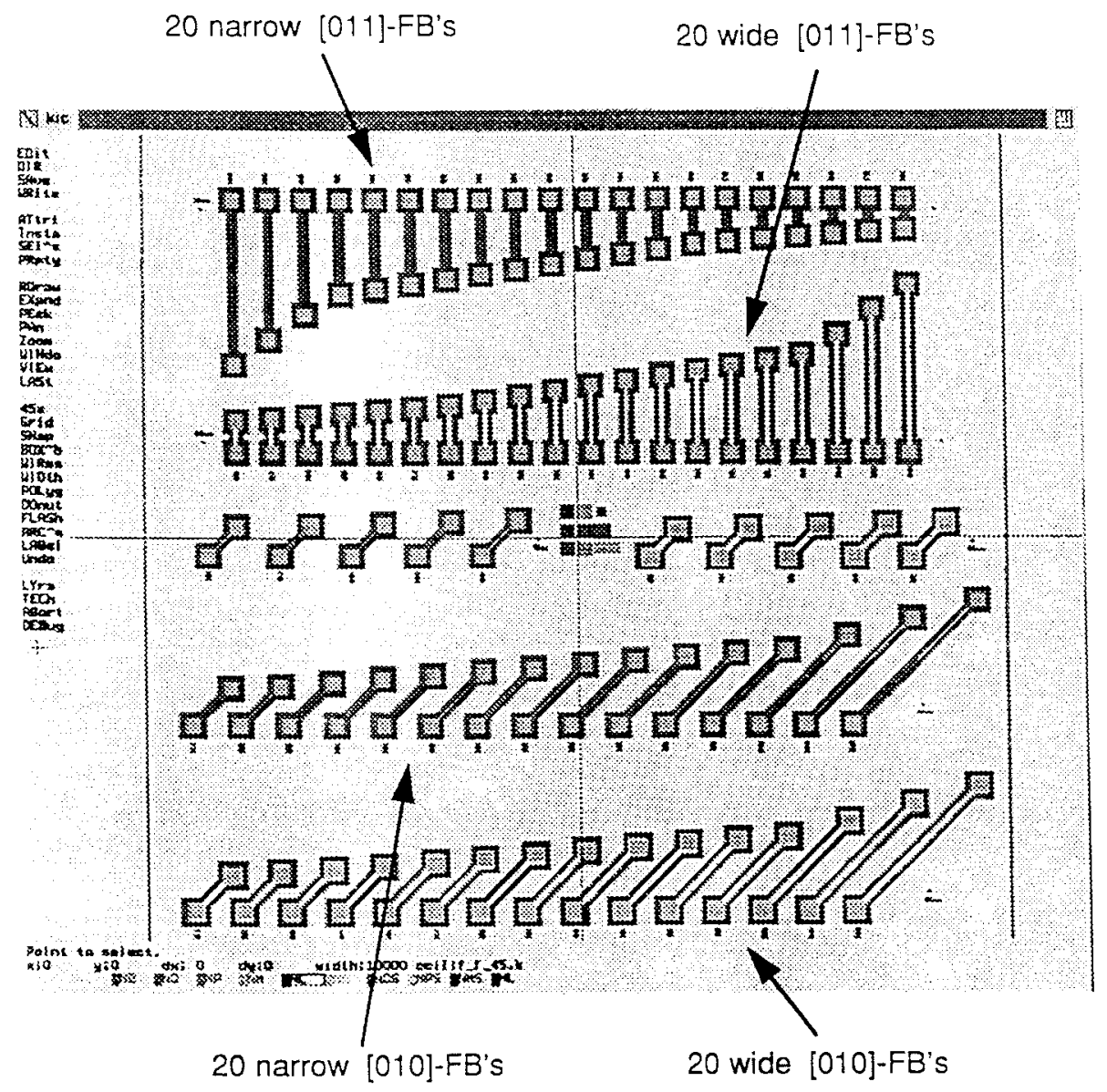

Fig. 10. FB MTEST mask layout. There are a total of $80 \mathrm{FB}$ test structures, including two beamwidth splits $(10$ and $50 \mu \mathrm{m})$ and two crystal orientation splits ([011] and [010]), as shown above. The beamlengths range from 150 to $1500 \mu \mathrm{m}$.

TABLE VII

Extracted $S$ ANd $B$ PARAMETERS ANd Material PROPERTIES FOR FB'S FROM MTEST-03 TEST WAFER

\begin{tabular}{|c|c|c|}
\hline B-parameter & $\begin{array}{c}{[011] \text { case }} \\
\left(\times 10^{-24} \mathrm{~Pa}^{6}\right)\end{array}$ & $\begin{array}{c}{[010] \text { case }} \\
\left.\left(\times 10^{-24} \mathrm{~Pa}^{6}\right)^{6}\right)\end{array}$ \\
\hline$B$ & $4.74 \pm 0.79$ & $4.06 \pm 0.63$ \\
\hline $\bar{B}$ & $4.94 \pm 0.32$ & $4.06 \pm 0.26$ \\
\hline$B^{\prime}$ & $4.95 \pm 0.14$ & $4.06 \pm 0.06$ \\
\hline
\end{tabular}

\begin{tabular}{|c|c|c|}
\hline S-parameter & $\begin{array}{c}{[011] \text { case }} \\
\left(\times 10^{-17} \mathrm{~Pa}^{4}\right)\end{array}$ & $\begin{array}{c}{[010] \text { case }} \\
\left(\times 10^{-17} \mathrm{~Pa}^{4} \mathrm{~m}^{4}\right)\end{array}$ \\
\hline \hline$S$ & $3.5 \pm 1.3$ & $3.5 \pm 1.9$ \\
\hline $\bar{S}$ & $3.5 \pm 0.5$ & $3.5 \pm 0.8$ \\
\hline$S^{\prime}$ & $3.5 \pm 0.5$ & $3.5 \pm 0.8$ \\
\hline
\end{tabular}

\begin{tabular}{|c|c|c|}
\hline Material Property & {$[011]$ case } & {$[010]$ case } \\
\hline \hline$\tilde{E}$ & $168 \pm 6 \mathrm{GPa}$ & $138 \pm 4 \mathrm{GPa}$ \\
\hline$\tilde{\sigma}$ & $10 \pm 1 \mathrm{MPa}$ & $10 \pm 2 \mathrm{MPa}$ \\
\hline
\end{tabular}

before scaling for thickness variation, while $S^{\prime}$ and $B^{\prime}$ are the averages after scaling. Note the improvement in standard error, which results from removal of thickness-correlated variation.

Finally, we extract material properties from the scaled mean values, $S_{0}^{\prime}$ and $B_{0}^{\prime}$, with results given in Table VII. The literature values for the plate modulii, $\tilde{E}_{[011]}$ and $\tilde{E}_{[010]}$, where $\tilde{E}=E /\left(1-\nu^{2}\right)$, of single-crystal silicon are 170 and 141 GPa, respectively [9], which are in excellent agreement $(\sim 2 \%)$ with the results in Table VII. Also, note that the statistical uncertainties in the plate modulus means in Table VII are less than $4 \%$, which is consistent with the excellent agreement found and provides a good indication of the overall validity of the M-Test approach.

The effective residual stress, $10 \mathrm{MPa}$, was expected to be small and turns out to be right at the edge of the minimum resolvable stress for this design. Most of the test structures had lengths or radii, which were within the bending-dominated regime. This was an "off-center" M-Test design, shifted toward the bending-dominated side.

\section{MTEST-03 CB and CD Results Summary}

1) $C B$ Results: Similar to the FB results above, $B$ parameter results were obtained from eight wide $\mathrm{CB}$ test structures from each of seven CB die from MTEST-03 for both the [011] and [010] crystal orientation cases [15]. (Note, there is no $S$ parameter data in the case of CB's since a CB has no residual stress.) Due to the aforementioned overetch that occurred on MTEST-03, the tips of the CB's and the substrate around the edges of the beam were affected, making precise determination of the effective gaps impossible. Therefore, precise material property extraction of $\tilde{E}$ was impossible. However, it was 
noted that the ratio of the CB's mean $B$ values, $\bar{B}_{[011]} / \bar{B}_{[010]}$, should agree with that of the FB's and the $\tilde{E}_{[011]-\mathrm{Si}} / \tilde{E}_{[010]-\mathrm{Si}}$ ratio from the literature. For the MTEST-03 CB's, we found

$$
\frac{\bar{B}_{[011]}}{\bar{B}_{[010]}}=\frac{6.03 \times 10^{-24} \mathrm{~Pa}-\mathrm{m}^{6}}{4.98 \times 10^{-24} \mathrm{~Pa}-\mathrm{m}^{6}}=1.21 \text {. }
$$

This compares very well to the values of 1.22 found for FB's and 1.21 from the literature [9].

2) CD Results: $S$ and $B$ parameter results were obtained from $14 \mathrm{CD}$ test structures from each of seven $\mathrm{CD}$ die from MTEST-03 [15]. Correlation checking between $S$ or $B$ and thickness or gap could not be done since no metrology data was done on any of the $\mathrm{CD}$ die. Therefore, a less precise set of material properties was extracted directly from the mean $B$ and $S$ values as follows:

$$
\begin{aligned}
& \tilde{E}=\frac{E}{1-\nu^{2}}=\frac{\bar{B}}{t_{0}^{3} g_{0}^{3}}=152 \pm 12 \mathrm{GPa} \\
& \tilde{\sigma}=\sigma_{0}=\frac{\bar{S}}{t_{0} g_{0}^{3}}=6.8 \pm 0.7 \mathrm{MPa} .
\end{aligned}
$$

The mean value of the extracted plate modulus (152 GPa) agrees with theory (155 GPa) [15]. (Note that "theory" here indicates that, at least for modeling displacements of this kind of structure, $155 \mathrm{GPa}$ serves well as an effective isotropic plate modulus of $\langle 100\rangle$ silicon.) The residual stress value falls in the expected range (1-10 Mpa) and is, effectively, at the limit of detection for this design.

\section{CONCLUSION}

Through quantitative modeling and improved experimental procedures, this work has demonstrated a methodology based on electrostatic pull-in of geometrically ideal electromechanical test structures (including CB's, FB's, and clamped CD's), which is now sufficiently accurate and robust to be used in both MEMS process development and manufacturing at the wafer level.

An important question is how to adapt the method to processes, which may not produce such ideal geometry or which may have compressive stress and/or compliant supports for the structures. All of these cause problems, but the problems are not necessarily insurmountable. Gupta [20] has already demonstrated that the models presented here can be readily modified to account for beams with a trapezoidal cross section (instead of ideal prismatic) and for such process artifacts as beam undercut during release etch, provided one is willing to make the geometric measurements needed to support the modeling. Furthermore, the method is now being successfully applied to structures with high residual stress and compliant supports [21], [22], at least for the purposes of monitoring process uniformity and repeatability. Quantitative extraction of residual stress and elastic moduli in the presence of stress gradients and residual compressive stresses has not yet been rigorously demonstrated, but there appears to be no fundamental reason why it should not be possible with sufficiently expanded models to account for such effects as support compliance, substrate curvature, and built-in imposed bending moments due to stress gradients.
Two major aspects of the work presented here are the design guidelines for how to apply M-Test to new processes, which generate approximately ideal structures, and a constructive procedure for how to expand the method to new processes and less ideal structures by showing how to build quantitatively reliable closed-form pull-in models based on meaningful intermediate parameters ( $S$ and $B$ in the case presented here) from which material properties can ultimately be extracted.

\section{ACKNOWLEDGMENT}

This research was carried out using the computer and process facilities of the Microsystems Technology Laboratories at MIT. The authors would like to extend special thanks to C. Hsu and Prof. M. Schmidt for providing the experimental test wafers and thanks to R. Gupta and Dr. J. Gilbert for valuable technical discussions.

\section{REFERENCES}

[1] S. D. Senturia, "Microfabricated structures for the measurement of mechanical properties and adhesion," in Proc. Transducers 87, Tokyo, Japan, pp. 11-16.

[2] K. E. Petersen, "Dynamic micromechanics on silicon: Techniques and devices," IEEE Trans. Electron Devices, vol. ED-25, no. 10, pp. 1241-1250, 1978.

[3] H. A. C. Tilmans, "Micro-mechanical sensors using encapsulated builtin resonant strain gauges," Ph.D. dissertation, MESA Research Instit. Univ. Twente, Enschede, The Netherlands, 1993.

[4] F. Maseeh, M. A. Schmidt, M. G. Allen, and S. D. Senturia, "Calibrated measurements of elastic limit, modulus, and the residual stress of thin films using micromachined suspended structures," in IEEE Solid State Sensor and Actuator Workshop, Hilton Head Island, SC, June 6-9, 1988, pp. 84-87.

[5] S. Wang, S. Crary, and K. Najafi, "Electronic determination of the modulus of elasticity and intrinsic stress of thin films using capacitive bridges," in Mat. Res. Soc. Symp. Proc., 1992, pp. 203-208.

[6] J. A. Schweitz, "Mechanical characterization of thin films by micromechanical techniques," MRS Bull., pp. 34-45, July 1992.

[7] M. G. Allen, M. Mehregany, R. T. Howe, and S. D. Senturia, "Microfabricated structures for the in-situ measurement of residual stress, Young's modulus, and ultimate strain of thin films," Appl. Phys. Lett., vol. 51, pp. 241-243, 1987.

[8] K. Najafi and K. Suzuki, "A novel technique and structure for the measurement of intrinsic stress and Young's modulus of thin films," in Proc. MEMS 89, Salt Lake City, UT, pp. 96-97.

[9] W. A. Brantley, "Calculated elastic constants for stress problems associated with semiconductor devices," J. Appl. Phys., vol. 44, no. 1, pp. 534-535, 1973.

[10] J. R. Gilbert, P. Osterberg, R. Harris, D. Ouma, A. Pfajfer, J. White, and S. D. Senturia, "Implementation of a MEMCAD system for electrostatic and mechanical analysis of complex structures from mask descriptions," in Proc. MEMS 93, Fort Lauderdale, FL, pp. 207-212.

[11] J. R. Gilbert, R. Legtenberg, and S. D. Senturia, "3D coupled electromechanics for MEMS: Applications of CoSolve-EM," in Proc. MEMS 95, Amsterdam, The Netherlands, pp. 122-127.

[12] C. H. Hsu and M. A. Schmidt, "Micromachined structures fabricated using wafer-bonded sealed cavity process," in Proc. 1994 Solid-State Sensor and Actuator Workshop, Hilton Head, SC, pp. 151-155.

[13] P. Osterberg, R. Gupta, J. Gilbert, and S. Senturia, "Quantitative models for the measurement of residual stress, poisson ratio, and Young's modulus using electrostatic pull-in of beams and diaphragms," in Proc. 1994 Solid-State Sensor and Actuator Workshop, Hilton Head, SC, pp. 184-188.

[14] P. Osterberg, H. Yie, X. Cai, J. White, and S. Senturia, "Self-consistent simulation and modeling of electrostatically deformed diaphragms," in Proc. MEMS 94, Oiso, Japan, pp. 28-32.

[15] P. M. Osterberg, "Electrostatically actuated microelectromechancial test structures for material property measurement," Ph.D. dissertation, Mass. Instit. Tech., Cambridge, MA, 1995.

[16] W. C. Young, Roark's Formulas for Stress and Strain. New York: McGraw-Hill, 1989, pp. 176-177. 
[17] S. Timoshenko, Theory of Plates and Shells. New York: McGraw-Hill, 1987, pp. 4-6, 118, 125

[18] MATLAB Version 4.2C, The Mathworks Inc., Natick, MA.

[19] Kaleidagraph Version 2.1.3, Synergy Software, Inc., Reading, PA.

[20] R. K. Gupta, C. H. Hsu, M. A. Schmidt, and S. D. Senturia, "Monitoring plasma over-etching of wafer-bonded microstructures," in Proc. Transducers 95, Stockholm, Sweden, pp. 269-272.

[21] R. K. Gupta, Ph.D. dissertation, Mass. Instit. Tech., Cambridge, in progress.

[22] P. Congdon and Texas Instruments, Inc., private communication, 1993-95.

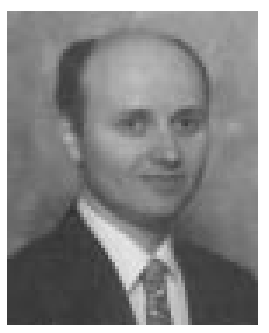

Peter M. Osterberg received the S.B. and S.M degrees in electrical engineering and computer science in 1980 and the Ph.D. degree in electrical engineering and computer science in 1995, all from the Massachusetts Institute of Technology (MIT), Cambridge. His Ph.D. research at MIT was in two areas: the simulation and computer-aided design of microelectromechanical systems (or MEMCAD) and MEMS material property measurement.

He worked for Texas Instruments, Inc., Dallas, TX, at their Central Research Laboratory from June 1978 to August 1979. There, he completed his S.B. and S.M. thesis work in the field of solid-state electromechanical spatial light modulators and deformable mirror displays (DMD's). He then worked for GTE, Inc., Needham, MA, from February 1980 to April 1986 as a VLSI Design/Test Engineering Manager in charge of an engineering group responsible for the design and test of semicustom CMOS ASIC chips for U.S. Government defense and communications applications. He then worked for Digital Equipment Corporation, Hudson, MA, from April 1986 to August 1991, where he was a VLSI Product Engineering Manager in charge of an engineering group responsible for the VLSI product engineering of several of DEC's CMOS full-custom and semicustom peripheral VAX computer chips. In 1996, he cofounded the MaxiMEM Limited consulting firm. His current research is in the area of MEMS-based cooling and power generation. He has been an Assistant Professor in the Electrical Engineering Department, University of Portland, OR, since 1996.

Dr. Osterberg is a Member of Tau Beta Pi and Eta Kappa Nu.

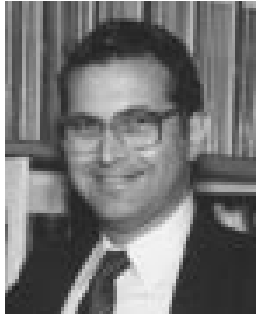

Stephen D. Senturia (F'93) received the B.A. degree in physics (summa cum laude) in 1961 and the Ph.D. degree in physics in 1966, both from Harvard University, Cambridge, MA.

He is presently the Barton L. Weller Professor of Electrical Engineering at the Massachusetts Institute of Technology (MIT), Cambridge. His recent research in solid-state sensors and actuators has included the development of the charge-flow transistor for use with thin-film sensor materials, the microdielectrometer for chemical reaction monitoring in resins, adhesives and plastics, and the silicon micromotor. His current principal research activities are the use of microfabricated structures, both for microsensor and microactuator applications, and for materials research, with special emphasis on CAD systems for design and simulation of microstructures (MEMCAD).

Dr. Senturia has been active in the program and organizing committees of the International Conference on Solid-State Sensors since Transducers '83, Delft, The Netherlands, and is Technical Program Chair for Transducers '97, Chicago, IL. He is a Trustee of the Transducer Research Foundation, which operates the Biannual North American Solid-State Sensors and Actuators Workshops. He served as Solid-State-Sensor Associate Editor of the IEEE Transactions on Electron Devices from 1985 to 1996 and was a Founding Associate Editor of the ASME/IEEE JouRnAL OF MiCROELECTROMECHANICAL SySTEMS. In 1982, he founded Micromet Instruments, Inc., and served on its Board of Directors until 1992 